\title{
Language production and working memory in classic galactosemia from a cognitive neuroscience perspective: future research directions
}

\author{
Inge Timmers • Job van den Hurk • Francesco Di Salle • \\ M. Estela Rubio-Gozalbo • Bernadette M. Jansma
}

Received: 31 March 2010 /Revised: 26 November 2010 / Accepted: 23 December 2010 /Published online: 3 February 2011

(C) The Author(s) 2011. This article is published with open access at Springerlink.com

\begin{abstract}
Most humans are social beings and we express our thoughts and feelings through language. In contrast to the ease with which we speak, the underlying cognitive and neural processes of language production are fairly complex and still little understood. In the hereditary metabolic disease classic galactosemia, failures in language production processes are among the most reported difficulties. It is unclear, however, what the underlying neural cause of this cognitive problem is. Modern brain imaging techniques allow us to look into the brain of a thinking patient online while she or he is performing a task, such as speaking. We can measure indirectly neural activity related to the output side of a process (e.g. articulation). But most importantly, we can look into the planning phase prior to an overt response, hence tapping into subcomponents of speech
\end{abstract}

Communicated by: Gerard T. Berry

Competing interest: None declared.

I. Timmers · J. van den Hurk · F. Di Salle $\cdot$ B. M. Jansma $(\square)$

Department of Cognitive Neuroscience,

Faculty of Psychology and Neuroscience, Maastricht University,

P. O. Box. 616, 6200 MD Maastricht, The Netherlands

e-mail: b.jansma@maastrichtuniversity.nl

I. Timmers $\cdot$ M. E. Rubio-Gozalbo

Department of Paediatrics, Maastricht University Medical Centre,

Maastricht, The Netherlands

J. van den Hurk $\cdot$ F. Di Salle $\cdot$ B. M. Jansma

Maastricht Brain Imaging Center (M-BIC),

Maastricht, The Netherlands

M. E. Rubio-Gozalbo

Laboratory of Genetic Metabolic Diseases,

Maastricht University Medical Centre,

Maastricht, The Netherlands planning. These components include verbal memory, intention to speak, and the planning of meaning, syntax, and phonology. This paper briefly introduces cognitive theories on language production and methods used in cognitive neuroscience. It reviews the possibilities of applying them in experimental paradigms to investigate language production and verbal memory in galactosemia.

\section{Introduction}

In normal healthy adults, speaking is fast, automatic, and nearly perfect. Only 1 word out of a 1000 goes wrong under normal circumstances. We speak to others, but we also speak to ourselves, continuously constructing streams of verbal thoughts. Only in the rare cases in which our speech production fails, we become aware of the speaking process and our errors. In contrast to the ease with which we speak, the underlying cognitive and neural process of language production is fairly complex and not fully understood yet. Especially when patients are impaired in speech and language, it is a scientific and clinical challenge to determine where in the language planning process the problem occurs. Exploring the nature and finding the cause of the problem, however, is of relevance for tailored treatment. Motor related problems should be treated differently than deficits of verbal working memory capacity, or the access to meaning and sounds of words, or syntactic skills.

Research from different fields such as Medicine, Psychology, Psycholinguistics, Neuropsychology, and Cognitive Neuroscience are working together to form a better understanding of the normal language system, but also of language disorders. Here we focus on the possibilities to join forces with regard to language impairments related to classic galactosemia. Classic galactosemia is a hereditary 
metabolic disease. There is a deficiency of the galactose1-phosphate uridyl transferase (GALT) enzyme, active in galactose metabolism (Holton et al. 2001). In the acute state, neonates present with lethargy, vomiting, diarrhea, failure to thrive, and jaundice. When treatment is started, a life-long galactose-restricted diet, the acute symptoms are relieved. However, despite diet the ovaries and the brain remain affected (Gubbels et al. 2008; Rubio-Gozalbo et al. 2010; Nelson et al. 1992; Dubroff et al. 2008). Osteopenia is also found (Panis et al. 2004), however, whether this is related to the disease itself or to acquired calcium and vitamin deficiencies (e.g. vitamin D and $\mathrm{K}$ ) is not yet clear. Among the cognitive problems that are experienced in classic galactosemia are lowered intelligence levels, memory problems and impaired language and speech (Antshel et al. 2004; Schweitzer et al. 1993; Waggoner et al. 1990). Most interestingly, and not at all understood, is that these cognitive impairments are developing in a child, despite the adequate dietary treatment. Abnormalities in speech and language have been estimated to be present in $38-88 \%$ of the patients (Hughes et al. 2009; Potter et al. 2008; Robertson et al. 2000; Schweitzer et al. 1993; Waggoner et al. 1990; Waisbren et al. 1983), although methods and samples differ in these studies. Problems are mainly said to be in expressive language. Receptive language is relatively unaffected, especially when intelligence problems are mild (Potter et al. 2008). There is still debate whether the problems are caused by cognitive failures or by a more sensory proprioceptive or motor failure, such as childhood apraxia of speech (CAS) (see also Potter et al. 2008, for a discussion on the CAS diagnosis). Related to CAS, it has been suggested that the observed impairment is an articulation deficit. This interpretation implies that the language disorder in these children is related to difficulties in the output phase of the language system. On the other hand, as the child acquires expressive language, the speech deficit could also be related to diverse stages in the neurocognitive preparation phase of speaking. According to language theories, these stages concern the selection of meaningful concepts to be expressed, their transfer into a meaningful grammatically well structured message, and phonological encoding of phonemes and syllables. In addition, third factors may play a role, such as general cognitive skills (IQ) or working memory as they may hamper planning and articulation directly or indirectly.

Until now, the cognitive problems in galactosemia have been described by expert speech therapists and neuropsychological testing (e.g. Antshel et al. 2004; Potter et al. 2008). These off-line instruments have the advantage of high feasibility. They are very informative, but also have the drawback of response bias, related to psychological factors and to response skills. A new and additional perspective in the study of galactosemia cognition comes from cognitive neuroscience. This field integrates knowledge on cognition with expertise in neuroscience and brain research. Using highly advanced techniques, brain activation related to cognitive function can be measured online, while the patient or healthy volunteer performs the task of interest. These methods allow us to track the neural activity related to the entire language planning process, from the intention to speak to articulation. By applying these techniques to classic galactosemia, one can objectively learn where and when in the neural system the difficulties occur along the information processing pathway. Findings could be useful for tailoring speech and other therapies for children with galactosemia. Now, speech therapy is primarily focused on improving articulation and less or not at all on language planning, such as practicing the construction of sentences. In a next step, the findings should contribute to the understanding of the underlying neural deficit. The detected brain regions of interest should be investigated at a micro level (e.g. in terms of metabolic effects per regions, or activation patterns at a cortical column level) to unravel the neural mechanisms that alter neural coding in galactosemia.

Towards a working hypothesis In 2007, we studied the medical records of 22 Dutch children with galactosemia (unpublished data). The cohort consisted of 11 males and 11 females, age at the time of testing ranged from $2 ; 1$ to 18;0. All children adhered to the diet, 13 had received speech therapy at one point in their lives $(59 \%)$ and 15 have (had) special educational programs (68\%). Reports from clinical experts were reviewed for relevant data on speech and language functioning. Next to articulatory problems (in $45 \%$ of the cases), these experts reported significant difficulties with sentence production in the patients (in $64 \%$ of the cases). Sentence production difficulties were described as making syntactic errors in sentences, producing sentences that were inappropriately short for their age, or producing incomplete sentences. Based on this observation, one working hypothesis is that patients with galactosemia suffer from a syntactical planning impairment, resulting in difficulties in sentence production. In addition to the language system itself, working memory is involved in language production as well and short sentences might be the result of such a verbal working memory problem (see Baddeley 1992; 2000, for working memory models; see Baddeley 2003, for the relation between language and working memory; Bock 1995; Bock and Levelt 1994, for syntactic production; Hagoort 2005, for the general psycholinguistic model and its link to underling brain function). Experimental paradigms can be applied to test whether the observed language impairment in classic galactosemia is related to malfunction within specific 
language expert systems or whether language deficits are a result of a limitation in verbal working memory.

\section{Methods used in cognitive neuroscience}

The cognitive neuroscience techniques can generally be classified based on temporal and spatial resolution. A high temporal resolution method is electroencephalography (EEG) and its derivative event related potential (ERP). In order to observe neural activity for one specific cognitive process, the EEG has to be time-locked to the onset of the stimulus or task by averaging the EEG signal in a time window around this moment of interest, resulting in the event related potential (ERP). Using ERP, one is able to look into a brain process of interest at a milliseconds time scale, which is very relevant for the study of cognition, in general a very fast information process. The planning of a word in a sentence, for example, takes about $600 \mathrm{~ms}$ from intention to articulation. Many ERP components (e.g. for perception, attention, memory, language planning, and execution) have been studied and characterized by their onset or peak latency, peak amplitude, and by their distribution across the scalp (Coles and Rugg 1995; Luck 2005). For an overview on language components, see Kutas and Schmitt (2003). High spatial resolution methods, on the other hand, such as functional Magnetic Resonance Imaging (fMRI), are able to locate networks involved in cognition (e.g. the language network), and to define functional distinct regions of interest within the network. In addition, they can provide information about structural and functional connectivity within the network. Diffusion tensor imaging (DTI) is a technique related to MRI that allows for tracking fibres of white matter, through which neural signals travel. Complementary to MRI, Magnetic Resonance Spectroscopy (MRS) is able to determine the concentration of brain metabolites, making it possible to investigate the biochemical profile of pathophysiological processes within areas imaged (Gujar et al. 2005). Together these methods deliver a great precision in unravelling the cognitive information processing within a neural network.

\section{Towards paradigms to study language production and working memory in galactosemia}

\section{Language production}

The boxes in Fig. 1 depict the cognitive "stages" of speaking. According to Levelt et al. $(1989 ; 1999)$ and Bock (1995), speaking begins with a planning of a message, i.e. the activation of a concept of what one wants to convey to a listener. This message can come into mind driven by various stimuli or ideas. After visual encoding of a scene or picture and after creating the concept (both still preverbal), the meaning of the message has to be encoded (semantic encoding). The next process is syntactic encoding where grammatical roles (e.g. subject versus object) and syntactical functions (e.g. tense) are assigned. These elements are assembled into a syntactic frame. In addition, various sorts of information about the word form are activated (e.g. morphemes such as pre- or suffixes). Finally, the phonological encoding can take place where the sound structure is determined in the form of phonological frames. When the planning of these elements is finished, the output is sent to the (pre)motor cortex to activate speech muscles to move the tongue and jaw, which allow proper articulation. In addition, we have the ability to monitor the process. This process screens the output from the speech production process for errors, and makes us overtly correct a slip. It can also work without us being aware of it within each of the expert systems (see Postma 2000, for a review). In a nutshell, Fig. 1 summarizes major findings from preceding research in language production. The numbers in the boxes of Fig. 1 refer to the time course, or speed, of information processing across stages. They reflect time windows in milliseconds starting from visual picture onset and are estimated via EEG. These numbers have recently been supported by Sahin et al. (2009) using invasive intracranial electrophysiological (ICE) recording.

The language system has also been described with regard to the brain regions sensitive to language production (see Indefrey and Levelt 2004, for a meta-analysis). The coloured regions superimposed on a standard brain in Fig. 1 depict the language system. As can be seen in this figure, the areas involved in language production are widespread. Parts of the temporal lobe are for instance involved in error monitoring and semantics. The parietal area (inferior parietal gyrus) is implicated in phonology. However, Broca's area (or BA 44/45) and the inferior frontal gyrus (IFG) have been found to be involved in semantics, syntax and phonology processes (see overview in Hagoort 2005; Sahin et al. 2009). It has been suggested that the left IFG is the site where the assembly or unification takes place after the relevant information has been recruited from other brain areas (Hagoort 2005), while the information is recruited from mid temporal areas (Snijders et al. 2009). The information is assumed to travel from the temporoparietal areas through the arcuate fasciculus (i.e. a neural pathway of white matter fibers) to the frontal areas (Catani and Mesulam 2008; Rilling et al. 2008).

In order to study language production in classic galactosemia from this perspective, we propose example paradigms with focus on core processes, such as conceptualization, syntactical encoding and verbal working memory. A summary 


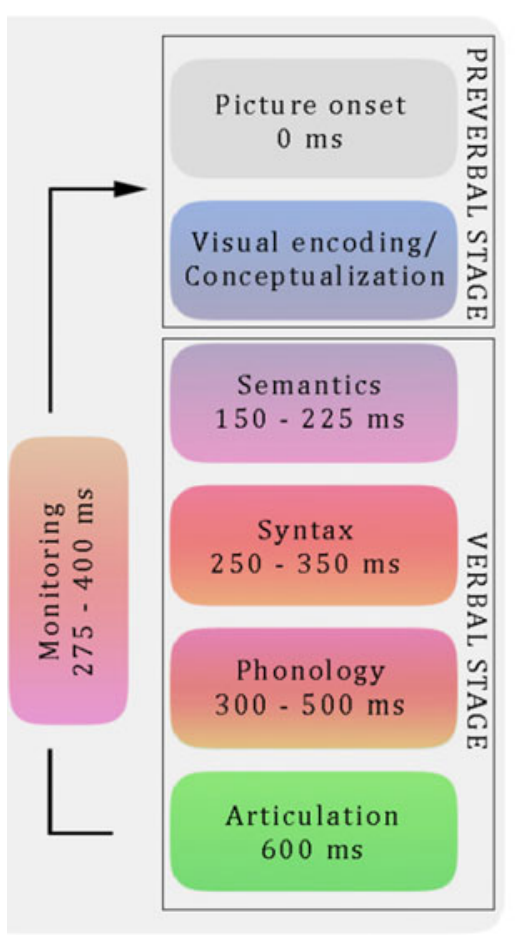

Fig. 1 Speech production model. Displayed are cognitive stages (left) and brain areas (right) involved in language production. The numbers in the boxes represent estimates of temporal encoding for each type of information. After picture presentation $(0 \mathrm{~ms})$, the visual system encodes the stimulus and activates a preverbal concept. The appropriate lexical entries are selected (150-225 ms, medial temporal gyrus (d)). The next stage involves syntactic encoding (left inferior frontal gyrus (IFG), taking place around $250-350 \mathrm{~ms}$ post stimulus (a)). Finally, phonological encoding takes place (300-500 ms, posterior superior temporal gyrus, angular gyrus (c)). The message is then

of the paradigms to be used is given in Table 1. This is a novel multidisciplinary approach that aims to provide new insights in the cognitive pathophysiological processes in galactosemia.

Observed differences (as compared to healthy subjects) in an experiment that taps conceptualization could be interpreted as a conceptualization deficit in the patient group. As conceptualization is input for further language encoding stages, the deficit in turn can cause subsequent problems in following stages. A lack of effect during a paradigm tapping conceptualization means that the observed language deficit in the patients may be attributed to later processing stages, after conceptualization took place. A difference in neural activity (as compared to healthy subjects) during a semantic encoding paradigm would point to impaired semantic processing in the patients. However, one can conclude a semantic deficit only in case the conceptualization account is ruled out for reasons expressed above. No differences between the groups with regard to semantic planning would indicate proper semantic encoding. Using this approach the different stages of language can be tested. One should keep in mind, finally, that any presumably assembled in the left IFG. After all planning has taken place, the finished speech plan is sent to (pre-) motor areas (b) to be prepared for articulation. An online self-monitoring feedback loop (275 - $400 \mathrm{~ms}$, superior temporal gyrus (e)) is capable of keeping track of the speech production process and intervenes if required. It has to be noted that boxes or stages are for display purpose only. Speech production does not involve encapsulated modules, but involves several brain regions that interact in a cascading manner. (Model adapted from Indefrey and Levelt 2004, plus recent temporal information, for example Sahin et al. 2009)

suggested impairment in a specific language process in galactosemia, necessitates excluding a verbal working memory deficit. There is a close interaction between language production systems and verbal working memory. In the context of galactosemia, it has to be investigated whether patients with galactosemia have difficulties with either one of these functions or perhaps with both. However, it is very difficult to disentangle what is attributable to an inability to maintain verbal information, for instance during syntactical encoding, or to an inability of syntactical encoding per se.

Furthermore, it should be noted that the translation of experimental paradigms from healthy study subjects to patients with galactosemia is not straightforward. As was described, many patients have a low intelligence and are, a priori, expected to have difficulties with mastering complex paradigms. Therefore, the paradigms have to be simplified such that even the lower functioning patients can perform them, or these patients should be excluded from the study group. In practice, there should be a balance between designing relatively simple paradigms and recruiting 
Table 1 Overview of example paradigms to study language production and working memory difficulties in galactosemia. An overview is given of example paradigms, and the concept of which they are based, to study the different language production and working memory stages. By implementing these paradigms it can be examined whether there is a difference in the time course of information processing (EEG/ERP) and in the neural correlates (fMRI) of these stages between galactosemia patients and healthy volunteers

\begin{tabular}{|c|c|}
\hline Cognitive stage & Concept \\
\hline Conceptualization & $\begin{array}{l}\text { People prefer a chronological order } \\
\text { when planning their sentence (e.g. } \\
\text { "After I saw my favourite meal, I } \\
\text { became hungry.") instead of a non- } \\
\text { chronological or reversed order (e.g. } \\
\text { "Before I became hungry, I saw my } \\
\text { favourite meal."). This is presumably } \\
\text { because non-chronologically ordered } \\
\text { sentences require a higher working } \\
\text { memory demand (Munte et al. 1998) }\end{array}$ \\
\hline
\end{tabular}

Semantics

The picture-word-interference (PWI) paradigm designed by Glaser and Düngelhoff (1984) based on the Stroop effect (Stroop 1935).

Syntax

Phonology

The picture-word-interference (PWI) paradigm designed by Glaser and Düngelhoff (1984).

Articulation

Verbal working memory

\section{Example paradigm}

Conceptualization conditions in which easy ("After") and difficult ("Before") sentences have to be produced are compared.

Subjects see a picture and see or hear an irrelevant word at the same time. They are asked to ignore this distractor and to name the picture. In case a picture of, for example, a dog is presented with the word cat, naming of "dog" is hampered due to semantic interference.

Based on animated visual scenes, overt sentence production with varying levels of syntax (easy, medium, complex) can be elicited. The conditions are compared.

Subjects see a picture and see or hear an irrelevant word at the same time. They are asked to ignore this distractor and to name the picture. In case a picture of, for example, a duck is presented with the word "dusk" (orthographically related), naming of "duck" is facilitated due to phonological relatedness.

Synchronized syllable repetitions Several kinds of tasks: overt speech by reading a passage from a book; the phonation of a monotone vowel; lip movements and tongue movements (both without actual vocalization), to separate vocalisation from articulation

Processing easy ("After") and difficult ("Before") sentences, and comparing the conditions. Interestingly, individuals with higher verbal working memory span showed a greater difference between the two conditions.

\section{References}

EEG/ERP (Marek et al. 2007) fMRI

(Zheng et al. accepted pending revision).

EEG/ERP (Hirschfeld et al. 2008) fMRI (de Zubicaray et al. 2001; 2006)

PET (Indefrey et al. 2001; 2003) Other approaches: Intracranial electrophysiology (ICE) (Sahin et al. (2009)

fMRI (de Zubicaray et al. 2002) Other approaches: Behavioural analyses (see Meyer 1992, for an overview) EEG/ERP (see meta-analysis of Indefrey and Levelt 2004)

fMRI (Riecker et al. 2005) fMRI (Brown et al. 2009; Brown et al. 2008)

EEG/ERP (Munte et al. 1998). when planning their sentence (e.g. "After I saw my favourite meal, I became hungry.") instead of a nonchronological or reversed order (e.g. "Before I became hungry, I saw my favourite meal.").
Of special interest in language production for galactosemia is the study of syntactic encoding. Our group has performed an ERP study using an adapted version of the Indefrey et al. experiment $(2001 ; 2003)$ in a group of 20 patients with intellectual abilities that allow executing the paradigms. When patients with a too low IQ (lower than 80) are excluded, the paradigms described in Table 1 all are expected to be feasible in the patient group. 
healthy subjects and in 9 adult galactosemia patients. The aim was to identify the ERP components related to syntactical encoding. Preliminary results indicate components of interest in our ERP data comparable to the findings of Sahin et al's ICE study, where distinct neural activity for semantic, grammatical and phonological encoding was found at around 200, 320 and $450 \mathrm{~ms}$ after target word onset, respectively, at the place of the electrode inserts (BA44/45 or Broca's area). A descriptive comparison of the
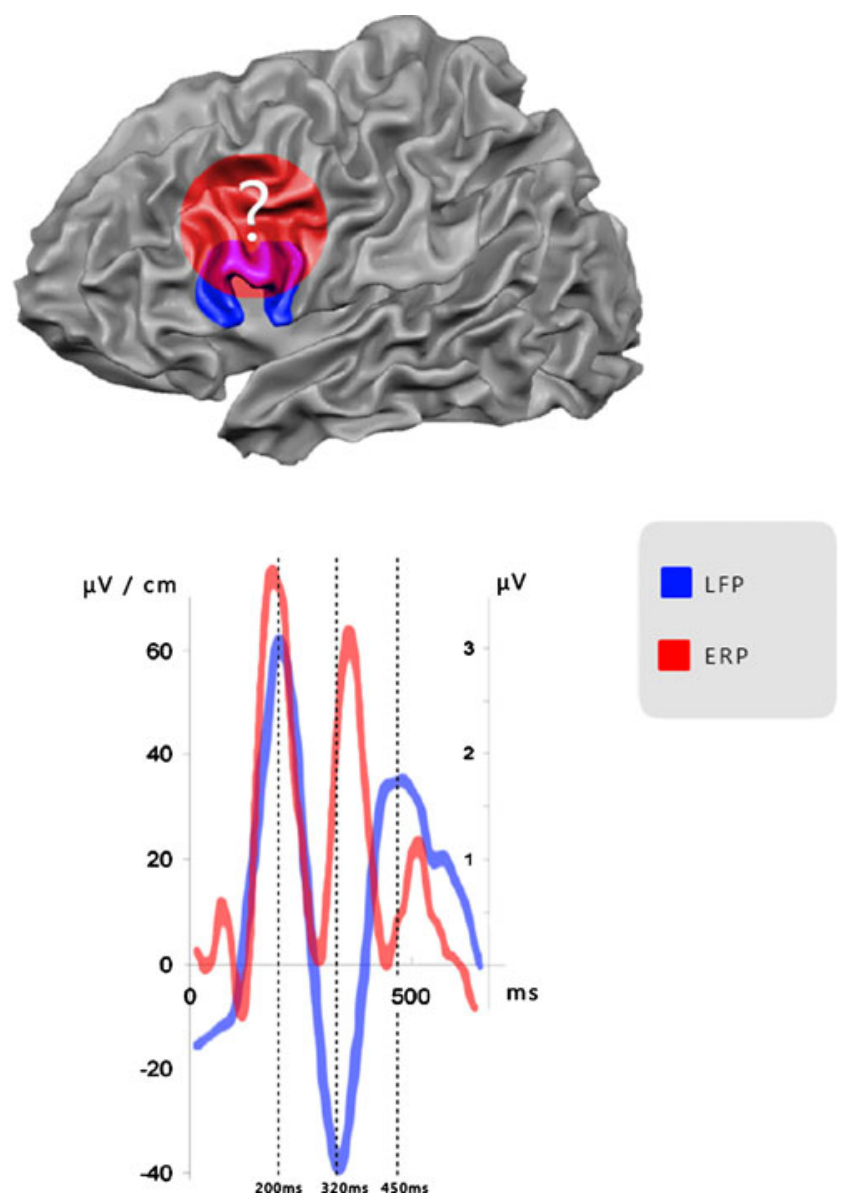

Fig. 2 Local field potentials (LFPs) versus ERPs during syntactical encoding. A descriptive comparison is made between the intracranial local field potentials of Sahin et al. (2009) and the extracranial EEG/ ERP study of our group. Both studies investigated the brain's response to the encoding of syntax. Sahin et al. instructed their participants to make grammatical inflections while our participants were asked to utter a complete sentence in response to an animated scene. Lower panel: Overlay of average LFP and ERP within the same time scale. Interestingly, despite the differences in the method and in the syntactic task, the morphology of the waveforms is strikingly similar in the target peak latencies $(200,320,450 \mathrm{~ms})$. Granting the assumption that peaks in LFP and ERP signal reflect maximal neural activity, this descriptive comparison suggests common aspects in the two signal types for language encoding. Upper panel: The brain area depicted in blue represents Broca's area, i.e. the location of the intracranial recording. The red circle reflects the presumed source of the EEG data (in correspondence with the PET study results of Indefrey et al. 2001; 2003, using the same paradigm). The EEG source still has to be confirmed data can be found in Fig. 2. Further, our findings support the claim that the IFG is not only involved in syntax (as was found by the PET study of Indefrey et al. 2001; 2003), but also in conceptualization and/or semantic processing. For galactosemia research, this means that the IFG is an area of special interest for further investigations. Preliminary results in the nine galactosemia adults suggest that language production processes in galactosemia might be delayed (i.e. delayed onset latencies of the waveforms) in addition to amplitude differences.

\section{Working memory}

The most widely used model of working memory comes from Baddeley (1992; 2000). In this model, working memory consists of multiple components (e.g. a central executive supervisory system; and an episodic buffer, a system proposed to link information forming one unified whole, such as in a movie scene). Working memory components have been described in terms of brain areas and networks (e.g. Cabeza and Nyberg 2000).

So far, not much is known on syntactic verbal memory. A suggestion is that the inferior frontal gyrus (IFG or BA44) plays a relevant role in assembling strings of information may that be phonological, syntactic, or semantic - into a meaningful message (Hagoort 2005). If one compares the language production network (Fig. 1) with the working memory network (Fig. 3), one can see that there is an overlap, especially in the IFG. Similar to the proposed function of the IFG in language production (selection and assembly or unification), this area is proposed to be involved in selection, retrieving and maintaining verbal information (Martin and Chao 2001), with syntactic retrieval from mid temporal lobes (Snijders et al. 2009). This view suggests that there is a close link between the language and the verbal working memory system.

\section{Linking the multiple disciplines}

Although it is very important to specify where and when failures occur during language production and working memory performance in patients with classic galactosemia, it might be even more important to understand why they occur. To understand the changes or deficits in neural information processing, it is important to study the specific data transfer and metabolic processes that affect information processing in pre-specified brain regions of interest to see how these specific cognitive problems result from the deficiency in galactosemia.

The pathogenic mechanisms in classic galactosemia and how they result in the cognitive impairments despite diet, are not entirely clear. Possibly, the damage has already 


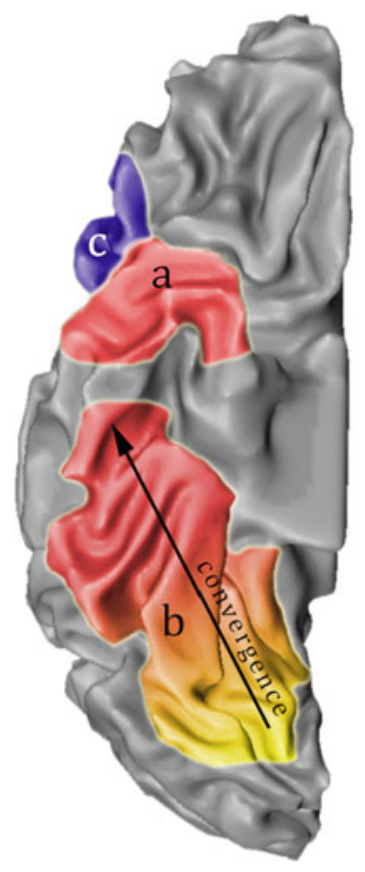

Fig. 3 Working memory model. The anterior temporal pole (a) is believed to play an important role in semantic memory retrieval and representation of specific semantic items. Regions in the fusiform gyrus (b) have proven to show differential responses to different categories of objects, converging in specificity from posterior to anterior regions. The inferior frontal gyrus (IFG) $(\mathbf{c}, \mathbf{d}, \mathbf{e})$ is involved in several (semantic) working memory related tasks: rehearsal (c), selection (d) and production (e). The lateral temporal cortex (f) is

occurred in utero, or in the first few days of life before the diet is introduced. Normally, galactose is converted into galactose-1-phosphate (Gal-1-P), further metabolized into glucose-1-phosphate by GALT (i.e. the Leloir pathway for galactose metabolism). Because of the GALT deficiency in galactosemia, there is an accumulation of Gal-1-P and galactitol, which is considered to be one of the most important factors in the pathophysiology. Apart from the toxicity of these metabolites for body tissue and organs, the accumulation is also believed to result in inositol abnormalities (see the paper by Gerry in this themed issue) and aberrant synthesis of glycoproteins and glycolipids, which are important building stones, for instance, for myelin. Myelin is a relevant component for functional data transfer between neurons and brain regions. Indeed, early anatomical MRI scans show abnormal white matter patterns in galactosemia patients, suggested to be due to abnormal myelin (Nelson et al. 1992), implying hampered data transfer. Both language production and working memory are carried out in specific networks (see Figs 1 and 3, respectively) that partly overlap with each other (both anatomically and possibly functionally). If there is a direct link between myelin degradation and brain function, one could expect that myelin is relatively more abnormal within these target areas. Studies with high resolution acquisition related to the perception of motion, of both biological (dorsal) and artificial (ventral) objects, and to lexical memory, whereas the posterior superior temporal gyrus (h) is the presumed region where phonologic loops are maintained. Finally, dorsolateral prefrontal cortex (dlPFC) (g) has an overall executive role in working memory tasks (after Cabeza et al. 2002; and Martin and Chao 2001). Obvious is the overlap of this memory network with the language network depicted in Fig. 1

and advanced analysing techniques will have to point out whether the specific functional areas are affected. One such approach could be Diffusion Tensor Imaging (DTI), a measure that is strongly linked to axonal and myelin integrity, and has proven a very sensible and early finding in several asymptomatic brain diseases (Nierenberg et al. 2005; Reading et al. 2005). In its more sophisticated application, DTI can even provide detailed information regarding anatomical connectivity, and will probably be able to indicate whether it diverges between galactosemia patients and healthy controls within the language and memory network. The studies that have already been done in galactosemia (Kaufman et al. 1995; Nelson et al. 1992) did not show clear regional differences, nor correlations with cognitive processes, based on the available data (low resolution 0.5-1.5 Tesla scanner). The availability of higher resolution scanner in the future allows investigating a functional link between myelin and cognition. However, one interesting finding of Nelson et al. is that about onethird of the patients had mild lateral ventricle enlargement and clustering of white matter lesions around the lateral ventricles. Fascinating about this report is that the arcuate fasciculus, the white matter bundle connecting temporoparietal language areas with the frontal language areas, is located alongside of the lateral ventricle. Abnormal myelin 
might affect the information flow within the networks and thus might affect language function. A target method of choice to investigate differences in such information flow within neural circuits, such as the arcuate fasciculus, in galactosemia patients versus healthy controls would be functional connectivity analysis.

Recently, it has been suggested that epigenetic factors may be involved in the pathology of galactosemia. Coman et al. (2010) studied gene expression profiles of four galactosemia patients. They identified several up- or downregulations in gene expressions in these patients. Genes involved in cell signaling pathways, such as the mitogen-activated protein kinase (MAPK) signaling and the calcium signaling pathway, both implicated in neural signaling processes, showed different expression patterns. The most dysregulated gene was Septin 4, of which the expression was decreased 85-fold. Septins are proteins that are involved in a large number of cellular functions, such as membrane dynamics, cytokinesis, vesicle trafficking, exocytosis, and apoptosis (Cao et al. 2009; Haller et al. 2005). Septin 4 (or SEPT4) proteins have been implicated in neurodegenerative diseases, such as Alzheimer's disease or Parkinson's disease. It is expressed in all human tissue, but shows an high expression in the brain (Haller et al. 2005). Further studies will be necessary to elucidate whether these genes are relevant for the origin of the chronic complications. One of the possibilities might be to use ultra high field imaging. With this method, the detection of proteins by producing specifically tailored contrast mechanisms, e.g. by the use of immunoconjugated magnetic nanoparticles (Hilger et al. 2007), might become possible in the future. This in turn might permit to quantify the density of specific substances, among which Septin 4, which can be linked to specific brain regions of functional interest, such as memory or language.

It would be intriguing to examine whether genes encoding for cognitive functions are differentially expressed in galactosemia. One such gene is the FOXP2 transcription factor gene, which has been implicated in speech and language disabilities (Enard et al. 2002; Fisher and Scharff 2009). Such a research would provide another missing link: the link between the genes and behavioural level. Ultimately and ideally, in the future a multidisciplinary approach in combining genes, gene expressions, protein imaging, and cognition in galactosemia should result in a working model that explains the cognitive complications observed in galactosemia.

\section{Conclusions}

A novel and innovative approach is suggested to bridge the gap in disciplines between the behavioural level on the one hand and the metabolic and cellular level on the other hand, in the context of the hereditary metabolic disease classic galactosemia. More specifically, we propose to study language production difficulties in galactosemic patients from the perspective of cognitive neuroscience and to correlate language behaviour with brain functions, connectivity, and metabolism. Experimental paradigms from language production research and highly advanced techniques allow studying the brain functions of a patient online. This approach has the major advantage that not only the output stage of language production can be studied, but the preceding planning stages as well. This is necessary as language production is a fairly complex process consisting of several subcomponents, referred to as conceptualization, semantic, syntactic, and phonological encoding, followed by articulation. It is highly relevant to learn exactly where and when in the cognitive and neural system of language the difficulties arise in classic galactosemia to be able to offer for example speech therapy in a tailored manner. In addition to language, verbal working memory is highly involved in language production processes and vice versa. Next to describing the cognitive deficits in terms of timing (ERP) and locations (fMRI), connectivity analysis and the analysis of metabolites related to functions are relevant to understand the underlying neural processing deficit.

The aim of applying these methods and accompanying paradigms in galactosemia research - an approach that has never been taken before - is to gain more insight in the cognitive pathophysiological processes in galactosemia.

Open Access This article is distributed under the terms of the Creative Commons Attribution Noncommercial License which permits any noncommercial use, distribution, and reproduction in any medium, provided the original author(s) and source are credited.

\section{References}

Antshel KM, Epstein IO, Waisbren SE (2004) Cognitive strengths and weaknesses in children and adolescents homozygous for the galactosemia Q188R mutation: a descriptive study. Neuropsychology 18(4):658-664

Baddeley A (1992) Working Memory. Science 255(5044):556-559

Baddeley A (2000) The episodic buffer: a new component of working memory? Trends Cogn Sci 4(11):417-423

Baddeley A (2003) Working memory and language: an overview. J Commun Disord 36(3):189-208

Bock K (1995) Sentence Production: From Mind to Mouth. In: Miller JL, Eimas PD (eds) Speech, Language, and Communication. Handbook of perception and cognition. Academic, San Diego, pp 181-216

Bock K, Levelt W (1994) Language Production: Grammatical Encoding. In: Gernsbacher MA (ed) Handbook of Psycholinguists. Academic Press, San Diego, pp 945-984

Brown S, Ngan E, Liotti M (2008) A larynx area in the human motor cortex. Cereb Cortex 18(4):837-845

Brown S, Laird AR, Pfordresher PQ, Thelen SM, Turkeltaub P, Liotti M (2009) The somatotopy of speech: Phonation and articulation in the human motor cortex. Brain Cogn 70(1):31-41 
Cabeza R, Nyberg L (2000) Imaging cognition II: An empirical review of 275 PET and fMRI studies. J Cogn Neurosci 12(1):1-47

Cabeza R, Dolcos F, Graham R, Nyberg L (2002) Similarities and differences in the neural correlates of episodic memory retrieval and working memory. Neuroimage 16(2):317-330

Cao LH, Yu WB, Wu YH, Yu L (2009) The evolution, complex structures and function of septin proteins. Cell Mol Life Sci 66 (20):3309-3323

Catani M, Mesulam M (2008) The arcuate fasciculus and the disconnection theme in language and aphasia: history and current state. Cortex 44(8):953-961

Coles M, Rugg M (1995) Event-related brain potentials: an introduction. In: Rugg M, Coles M (eds) Electrophysiology of Mind: Event-Related Brain Potentials and Cognition. Oxford University Press, New York, US, pp 1-26

Coman DJ, Murray DW, Byrne JC et al (2010) Galactosemia, a Single Gene Disorder With Epigenetic Consequences. Pediatr Res 67 (3):286-292

de Zubicaray GI, Wilson SJ, McMahon KL, Muthiah S (2001) The Semantic Interference Effect in the Picture-Word Paradigm: An Event-Related fMRI Study Employing Overt Responses. Hum Brain Mapp 14:218-227

de Zubicaray GI, McMahon KL, Eastburn MM, Wilson SJ (2002) Orthographic/Phonological Facilitation of Naming Responses in the Picture-Word Task: An Event-Related fMRI Study Using Overt Vocal Responding. Neuroimage 16:1084-1093

de Zubicaray GI, McMahon KL, Eastburn MM, Pringle A (2006) Topdown influences on lexical selection during spoken word production: A $4 \mathrm{~T}$ fMRI investigation of refractory effects in picture naming. Hum Brain Mapp 27(11):864-873

Dubroff JG, Ficicioglu C, Segal S, Wintering NA, Alavi A, Newberg AB (2008) FDG-PET findings in patients with galactosaemia. J Inherit Metab Dis 31(4):533-539

Enard W, Przeworski M, Fisher SE et al (2002) Molecular evolution of FOXP2, a gene involved in speech and language. Nature 418:869-872

Fisher SE, Scharff C (2009) FOXP2 as a molecular window into speech and language. Trends Genet 25(4):166-177

Glaser WR, Düngelhoff F-J (1984) The time course of pictureword interference. J Exp Psychol Hum Percept Perform 10 (5):640-654

Gubbels CS, Land JA, Rubio-Gozalbo ME (2008) Fertility and Impact of Pregnancies on the Mother and Child in Classic Galactosemia. Obstet Gynecol Surv 63(5):334-343

Gujar SK, Maheshwari S, Bjorkman-Burtscher I, Sundgren PC (2005) Magnetic resonance spectroscopy. J Neuroophthalmol 25 (3):217-226

Hagoort P (2005) On Broca, brain, and binding: a new framework. Trends Cogn Sci 9(9):416-423

Haller S, Radue EW, Erb M, Grodd W, Kircher T (2005) Overt sentence production in event-related fMRI. Neuropsychologia 43 (5): $807-814$

Hilger I, Trost R, Reichenbach JR et al (2007) MR imaging of Her$2 /$ neu protein using magnetic nanoparticles. Nanotechnology 18 : 135103

Hirschfeld G, Jansma B, Bolte J, Zwitserlood P (2008) Interference and facilitation in overt speech production investigated with event-related potentials. NeuroReport 19(12):1227-1230

Holton JB, Walter JH, Tyfield LA (2001) Galactosemia. In: Scriver CR, Childs B (eds) The metabolic \& molecular bases of inherited disease. McGraw-Hill, New York, pp 1553-1587

Hughes J, Ryan S, Lambert D et al (2009) Outcomes of Siblings with Classical Galactosemia. J Pediatr 154(5):721-726

Indefrey P, Levelt WJM (2004) The spatial and temporal signatures of word production components. Cognition 92(1-2): $101-144$
Indefrey P, Brown CM, Hellwig F et al (2001) A neural correlate of syntactic encoding during speech production. Proceedings of the National Academy of Sciences of the United States of America 98: 5933-5936

Indefrey P, Hellwig F, Herzog H, Seitz RJ, Hagoort P (2003) Neural responses to the production and comprehension of syntax in identical utterances. Brain Lang 89:312-319

Kaufman FR, McBride Chang C, Manis FR, Wolff JA, Nelson MD (1995) Cognitive functioning, neurologic status and brain imaging in classical galactosemia. Eur J Pediatr 154(7 Suppl 2): S2-S5

Kutas M, Schmitt BM (2003) Language in Microvolts. In: Banich MT, Mack M (eds) Mind, brain, and language: Multidisciplinary perspectives. Lawrence Erlbaum Associates, NJ, pp $171-209$

Levelt WJM (1989) Speaking: From Intention to Articulation. The MIT Press, Cambridge, MA

Levelt WJM, Roelofs AS, Meyer A (1999) A theory of lexical access in speech production. Behav Brain Sci 22(1):1-75

Luck SJ (2005) An Introduction to the Event-Related Potential Technique. MIT Press, Cambridge, MA

Marek A, Habets B, Jansma BM, Nager W, Munte TF (2007) Neural correlates of conceptualisation difficulty during the preparation of complex utterances. Aphasiology 21(12):1147-1156

Martin A, Chao LL (2001) Semantic memory and the brain: structure and processes. Curr Opin Neurobiol 11(2):194-201

Meyer AS (1992) Investigation of Phonological Encoding through Speech Error Analyses - Achievements, Limitations, and Alternatives. Cognition 42(1-3):181-211

Munte TF, Schiltz K, Kutas M (1998) When temporal terms belie conceptual order. Nature 395(6697):71-73

Nelson MD, Wolff JA, Cross CA, Donnell GN, Kaufman FR (1992) Galactosemia: Evaluation with MR Imaging. Radiology 184:255-261

Nierenberg J, Pomara N, Hoptman MJ, Sidtis JJ, Ardekani BA, Lim KO (2005) Abnormal white matter integrity in healthy apolipoprotein E epsilon4 carriers. NeuroReport 16:1369-1372

Panis B, Forget PP, van Kroonenburgh MJ et al (2004) Bone metabolism in galactosemia. Bone 35(4):982-987

Postma A (2000) Detection of errors during speech production: a review of speech monitoring models. Cognition 77(2):97-131

Potter NL, Lazarus JAC, Johnson JM, Steiner RD, Shriberg LD (2008) Correlates of language impairment in children with galactosaemia. J Inherit Metab Dis 31(4):524-532

Reading SA, Yassa MA, Bakker A et al (2005) Regional white matter change in pre-symptomatic Huntington's disease: a diffusion tensor imaging study. Psychiatry Res 140:55-62

Riecker A, Mathiak K, Wildgruber D et al (2005) fMRI reveals two distinct cerebral networks subserving speech motor control. Neurology 64(4):700-706

Rilling JK, Glasser MF, Preuss TM et al. (2008) The evolution of the arcuate fasciculus revealed with comparative DTI. Nat Neurosci $11: 426-428$

Robertson A, Singh RH, Guerrero NV, Hundley M, Elsas LJ (2000) Outcomes analysis of verbal dyspraxia in classic galactosemia. Genet Med 2(2):142-148

Rubio-Gozalbo ME, Gubbels CS, Bakker JA, Menheere PPCA, Wodzig WKWH, Land JA (2010) Gonadal function in male and female patients with classic galactosemia. Hum Reprod Update 16(2):177-188

Sahin NT, Pinker S, Cash SS, Schomer D, Halgren E (2009) Sequential Processing of Lexical, Grammatical, and Phonological Information Within Broca's Area. Science 326(5951): 445-449

Schweitzer S, Shin Y, Jakobs C, Brodehl J (1993) Long-term outcome in 134 patients with galactosemia. Eur J Pediatr 152:36-43 
Snijders TM, Vosse T, Kempen G, Van Berkum JJA, Petersson KM, Hagoort P (2009) Retrieval and Unification of Syntactic Structure in Sentence Comphrehension: an fMRI Study Using Word-Category Ambiguity. Cereb Cortex 19:1493-1503

Stroop JR (1935) Studies of interference in serial verbal reactions. J Exp Psychol 18:643-662
Waggoner DD, Buist NRM, Donnell GN (1990) Long-Term Prognosis in Galactosemia: Results of a Survey of 350 Cases. J Inherit Metab Dis $802-818$

Waisbren SE, Norman TR, Schnell RR, Levy HL (1983) Speech and language deficits in early-treated children with galactosemia. J Pediatr 102(1):75-77 